\title{
Vaginal flora morphotypic profiles and assessment of bacterial vaginosis in women at risk for HIV infection
}

\author{
Beth C Tohill', Charles M Heilig', Robert S Klein², Anne Rompalo³, \\ Susan Cu-Uvin ${ }^{4}$, William Brown ${ }^{5}$ and Ann Duerr ${ }^{1}$ \\ ${ }^{1}$ Centers for Disease Control and Prevention, National Center for Chronic Disease and Public Health \\ Prevention, Division of Nutrition and Physical Activity, Atlanta, GA, USA \\ ${ }^{2}$ Montefiore Medical Center, Albert Einstein College of Medicine, Bronx, NY, USA \\ ${ }^{3}$ Johns Hopkins University, Baltimore, MD, USA \\ ${ }^{4}$ Brown University, Providence, RI, USA \\ ${ }^{5}$ Wayne State University, Detroit, MI, USA
}

Specific morphotypic profiles of normal and abnormal vaginal flora, including bacterial vaginosis (BV), were characterized. A prospective study of 350 women yielded concurrent Gram-stain data and clinical assessment ( $n=3455$ visits). Microbiological profiles were constructed by Gram stain. Eight profile definitions were based on dichotomizing the levels of Lactobacillus, Gardnerella, and curved, Gram-negative bacillus (Mobiluncus) morphotypes. Of these, two were rare, and the other six demonstrated a graded association with the clinical components of BV. The proposed profiles from the Gram stain reflect the morphotypic categories describing vaginal flora that may enable clearer elucidation of gynecologic and obstetric outcomes in various populations.

Key words: BACTERIAL VAginosis; Genital InFECTIONS; GRAM StAin; GARDNERELLA VAGINALIS;

\section{INTRODUCTION}

Bacterial vaginosis (BV) is a pervasive and persistent problem among women worldwide. It is a condition or syndrome characterized by a shift in vaginal microflora, whereby hydrogen peroxide-producing Lactobacilli are replaced by an overgrowth of aerobic and anaerobic bacteria, thus upsetting the ecological balance of the vagina. Prevalence of $\mathrm{BV}$ in the United States ranges from $10 \%$ to $25 \%$ in gynecological and obstetric practices and up to $64 \%$ in populations in sexually transmitted disease (STD) clinics ${ }^{1,2}$. It is now known to carry potentially serious consequences including possible associations with an increased risk of pelvic inflammatory disease ${ }^{3}$ and preterm delivery ${ }^{4}$. BV may contribute to genital tract shedding of HIV in HIV-infected women $^{5}$, and it may also increase a woman's risk of acquisition of HIV and other STDs from an infected partner ${ }^{6}$. Therefore there are many adverse conditions that are possibly associated with the presence of BV.

The two common methods for diagnosing BV are assessment of clinical manifestations and identification of abnormalities in vaginal flora by

Correspondence to: Beth C. Tohill, Centers for Disease Control and Prevention, National Center for Chronic Disease and Public Health Prevention, Division of Nutrition and Physical Activity, 4770 Buford Highway MS K-34, Atlanta, GA 30341, USA.

Email: btohill@cdc.gov 
microscopic examination of Gram-stained slides of vaginal secretions ${ }^{7,8}$. Although physicians tend to use the clinical criteria for diagnosis ${ }^{9}$, the Gram stain method is the overwhelming method of choice in research settings and is endorsed by many as the gold standard ${ }^{8,10,11}$. Microflora are substantially heterogeneous whether within a normal, abnormal, or BV condition. This diagnostic uncertainty has implications for clinical management. In addition, this uncertainty may underlie the failure of studies of BV to find consistent associations with outcomes and may help explain why several interventional trials of BV treatment have had little impact on the proposed outcome.

We used data from US women who were evaluated by both clinical and laboratory diagnostic methods, to explore whether different constellations of vaginal flora morphotypes exhibit clinically meaningful distinctions. We categorized the morphotypes from vaginal Gram stain into homogeneous groups and correlated these profiles with clinical symptoms that constitute the clinical criteria for BV.

\section{METHODS}

Data were analyzed from a cohort of 350 HIVuninfected women who were followed in the Human Immunodeficiency Virus Epidemiological Research (HER) Study ${ }^{12}$, a multi-site prospective study of women aged 16-55 years with or at-risk for HIV infection. The HER study sites included clinical and academic institutions in four US communities. Recruitment, screening and enrollment began in April 1993, and followup for the HIV-uninfected women ended in September 1999. All participants were categorized as having had a history of injection drug use after 1985 or self-reported, high-risk sexual behavior according to defined risk criteria. The protocol was approved by institutional review boards at the four study sites and the US Centers for Disease Control and Prevention. All participants gave their informed consent to participate in the study.

The visits included a standardized face-to-face interview that gathered information on medical symptoms, illnesses, medical procedures, repro- ductive events, contraceptive use, insurance status, tobacco, alcohol, drug use, and sexual behaviors, as well as psychosocial and demographic factors. A standardized physical exam followed each interview. The gynecologic exam included screening for pregnancy, genital tract inflammation, vaginal discharge, and cervical dysplasia. The laboratory assessment included blood testing for HIV (enzyme-linked immunosorbent assay) and syphilis serology; Papanicolau smear; swab of the posterior vaginal fornix used to prepare a Gram-stained slide; saline and $\mathrm{KOH}$ wet mounts of vaginal secretions for Trichomonas vaginalis, clue cells, and yeast; vaginal $\mathrm{pH}$ assessment using indicator strips (Baxter S/P, Glendale, $\mathrm{CA}$; whiff-amine test to note if there was a release of a fishy odor after adding a drop of 10\% $\mathrm{KOH}$ to vaginal fluids; a vaginal culture for Candida and Trichomonas vaginalis; and an endocervical culture for Neisseria gonorrhea and an endocervical culture and direct fluorescent antibody test for Chlamydia trachomatis.

Bacterial vaginosis was diagnosed by two methods: Amsel's (or clinical) criteria and $\mathrm{Nu}-$ gent's (or laboratory) criteria. Clinical BV by Amsel's criteria ${ }^{13}$ assessed by research nurses was defined by the presence of three or four of the following signs: positive whiff-amine test, vaginal $\mathrm{pH}>4.7$, clue cells on wet mount, and abnormal vaginal discharge. The laboratory definition is constructed from Gram-stained scores for Lactobacillus, Gardnerella, and Mobiluncus morphotypes. All smears of the posterior vaginal fornix were air dried, fixed in methanol, and then shipped to the Detroit Medical Center University Laboratories, Detroit, MI, for Gram-staining. Under oil immersion ( $\times 1000$ magnification) each slide was examined and scored for the aforementioned morphotypes by a single technologist who was unaware of the clinical or HIV status of the study participants. Though there was not a second technologist reviewing the slides, this one technician provided consistency of the readings throughout the entire study period. Each of the three morphotype scores was quantified on a scale of 0 to 4 , and these three scores were combined according to Nugent's formula $\left.{ }^{8}\right)$ : Gardnerella score $+(4-$ Lactobacillus score $)+$ Mobiluncus score/ 2 , where fractions are rounded up. This formula 
yields a score of $0-10$. Under this formula, a score of 7-10 defines BV; 4-6 is abnormal vaginal flora; and $0-3$ is regarded as normal vaginal flora.

Eight morphotypic profiles were constructed by dichotomizing at the median both Lactobacillus and Gardnerella scores at $0-2$ or 3-4 and Mobiluncus at 0 or $1-4$, and using each possible combination of the three as one profile (Table 1).

This analysis considered data from HIV-uninfected women only, as there are differences in the natural history of BV in HIV-infected women ${ }^{14}$. Data analysis was restricted to women who had at least three consecutive visits in which they were assessed by both laboratory and clinical diagnostic methods (350 women; 3,455 study visits; median number of visits per woman was 10 , range 2 to 14). The frequency of the eight profiles, as well as the frequency of the two diagnostic methods within the profiles, was assessed. The profiles were cross-tabulated with Nugent scores, clinical BV, and the individual clinical signs.

\section{RESULTS}

The women included in this analysis had a mean age of 35 years, 54\% were African American, 50\% reported intravenous drug use since 1985, and $46 \%$ reported having more than five sex partners in the previous five years.

Of the eight morphotypic categories, only the six (A, C, D, F, G, and $\mathrm{H}$ ) that occurred with any appreciable frequency will be discussed (Table 1). Profile A fit neatly into the Nugent score of $0-3$, which is thought to represent normal vaginal flora. Profile $\mathrm{H}$ fits almost exactly into the Nugent score of $7-10$, which is classic BV. The other four profiles spread across Nugent's intermediate group (scores 4-6), usually considered as abnormal. These four profiles also overlap with the normal and BV Nugent categories.

The correlation of profiles with the frequency of laboratory $\mathrm{BV}$ shows a progressive increase in frequency of BV. For example, 48\% of women with Profile C fall into the abnormal Gram stain category; $85 \%$ and $83 \%$, respectively, of Profiles $\mathrm{D}$ and $\mathrm{F}$ fall into the abnormal flora category; $27 \%$ of Profile G falls into the abnormal category and the remaining $73 \%$ falls into the $\mathrm{BV}$ category. Although both clinical and laboratory BV become relatively more frequent among abnormal profiles, within individual profiles the two diagnostic modes demonstrate notable differences (Table 1). The more normal profile (A) was more likely to meet clinical BV criteria than lab BV criteria; the reverse was true for the more abnormal profiles $(G, H)$. Profile $C$ is a category representing a sparse flora predominantly seen after antibiotic treatment and/or estrogen deficiency. Additionally, 34\% of profile D (observed high Gardnerella), which would not be classified as BV by the Nugent scoring, had $\mathrm{BV}$ by the clinical definition. Profiles $\mathrm{D}$ and $\mathrm{F}$

Table I Make-up of the morphotypic profiles and the percentage of each within the range of the Nugent scoring, clinical bacterial vaginosis (BV) and lab BV

\begin{tabular}{|c|c|c|c|c|c|c|c|c|c|c|c|c|c|c|c|c|c|}
\hline & \multicolumn{3}{|c|}{ Morphotypes* } & \multirow{2}{*}{$\begin{array}{c}\text { Freq } \\
\mathrm{N}\end{array}$} & \multicolumn{11}{|c|}{ Percentage within each Nugent score } & \multirow{2}{*}{$\begin{array}{c}\text { Clin } B V^{\dagger} \\
\text { (\%) }\end{array}$} & \multirow{2}{*}{$\begin{array}{r}\text { Lab BV } \\
(\%)\end{array}$} \\
\hline & Lact & Gard & CGNB & & 0 & 1 & 2 & 3 & 4 & 5 & 6 & 7 & 8 & 9 & 10 & & \\
\hline$A$ & $3-4$ & $0-2$ & 0 & 1579 & 63 & 25 & 9 & 3 & & & & & & & & 2.5 & 0.0 \\
\hline B & $3-4$ & $0-2$ & $1-4$ & 2 & & 0 & 0 & 50 & 50 & 0 & & & & & & & \\
\hline C & $0-2$ & $0-2$ & 0 & 241 & & & 29 & 22 & 32 & 12 & 4 & & & & & 7.5 & 0.0 \\
\hline $\mathrm{D}$ & $3-4$ & $3-4$ & 0 & 192 & & & & 15 & 45 & 40 & & & & & & 34.4 & 0.0 \\
\hline$E$ & $0-2$ & $0-2$ & $1-4$ & 7 & & & & 0 & 14 & 43 & 29 & 14 & 0 & & & & \\
\hline $\mathrm{F}$ & $3-4$ & $3-4$ & $1-4$ & 46 & & & & & 15 & 33 & 35 & 17 & & & & 41.3 & 17.4 \\
\hline G & $0-2$ & $3-4$ & 0 & 650 & & & & & & 5 & 22 & 48 & 25 & & & 48.6 & 73.1 \\
\hline \multirow[t]{2}{*}{$\mathrm{H}$} & $0-2$ & $3-4$ & $1-4$ & 738 & & & & & & & I & II & 40 & 34 & 14 & 67.8 & 99.1 \\
\hline & & & & 3455 & & & & & & & & & & & & & \\
\hline
\end{tabular}

*Morphotypes include Lact $=$ Lactobacillus, Gard $=$ Gardnerella, and CGNB $=$ Mobiluncus

'Clinical BV as defined by Amsel's criteria of having 3 of the 4 clinical signs.

Laboratory BV as defined by Nugent score $>6$. 
represent an increase in Gardnerella without the loss of lactobacillus.

To further elucidate the clinical manifestations of each profile, the frequency of individual clinical signs was examined by profile (Figure 1). Profile C, which has low Lactobacillus but otherwise resembles Profile A, had few abnormal clinical signs except for a high frequency of abnormal vaginal $\mathrm{pH}$, Characteristic of a postantibiotic vaginal flora. Substantial changes in clinical spectrum were seen in profiles $\mathrm{D}$ and $\mathrm{F}$ which retained high Lactobacillus but also had Gardnerella or both Gardnerella and Mobiluncus morphotypes; each of the four clinical signs was present in at least $40 \%$ of the occurrences of these profiles. Abnormal clinical signs, particularly increased $\mathrm{pH}$, were further increased among profiles $\mathrm{G}$ and $\mathrm{H}$, which closely resemble classic $\mathrm{BV}$. The difference between profiles $\mathrm{D}$ and $\mathrm{F}$, the presence of Mobiluncus, does not appear to have a strong association with the presence of clinical signs. The same pattern holds when comparing profiles $\mathrm{G}$ and $\mathrm{H}$, even though Lactobacillus levels are low. Comparing profiles $\mathrm{G}$ and $\mathrm{H}$ to $\mathrm{D}$ and $\mathrm{F}$ reveals an increase in elevated $\mathrm{pH}$, again associated with a loss of Lactobacillus.

\section{DISCUSSION}

Our morphotypic profiles used the three morphotypes used to diagnose BV by the Gram stain (Lactobacillus, Gardnerella, and Mobiluncus) characterized the spectrum of vaginal flora in this cohort. In comparison of the profiles to clinical $\mathrm{BV}$ diagnosis, the percentage of visits with clinical $\mathrm{BV}$ as well as the frequency of the individual clinical signs, increased as the vaginal flora shifted away from normal/healthy. The large increases in frequency of elevated $\mathrm{pH}$ seen in profiles $\mathrm{C}, \mathrm{G}$, and $\mathrm{H}$ illustrate the central role of Lactobacillus in maintaining low vaginal $\mathrm{pH}$. The increase in clinical signs in profiles $\mathrm{D}$ and $\mathrm{F}$ suggests that the presence of high Gardnerella plays an important role in causing clinical signs, even in the presence of high levels of Lactobacillus.

In addition, the defined morphotypic profiles clearly correlate with clinical signs. These associations, however, were not absolute. For example, the full range of clinical signs was seen in women with Gardnerella in both the presence and absence of Lactobacillus, although clinical signs were more common in the latter group of women. At least some women within each morphotypic profile

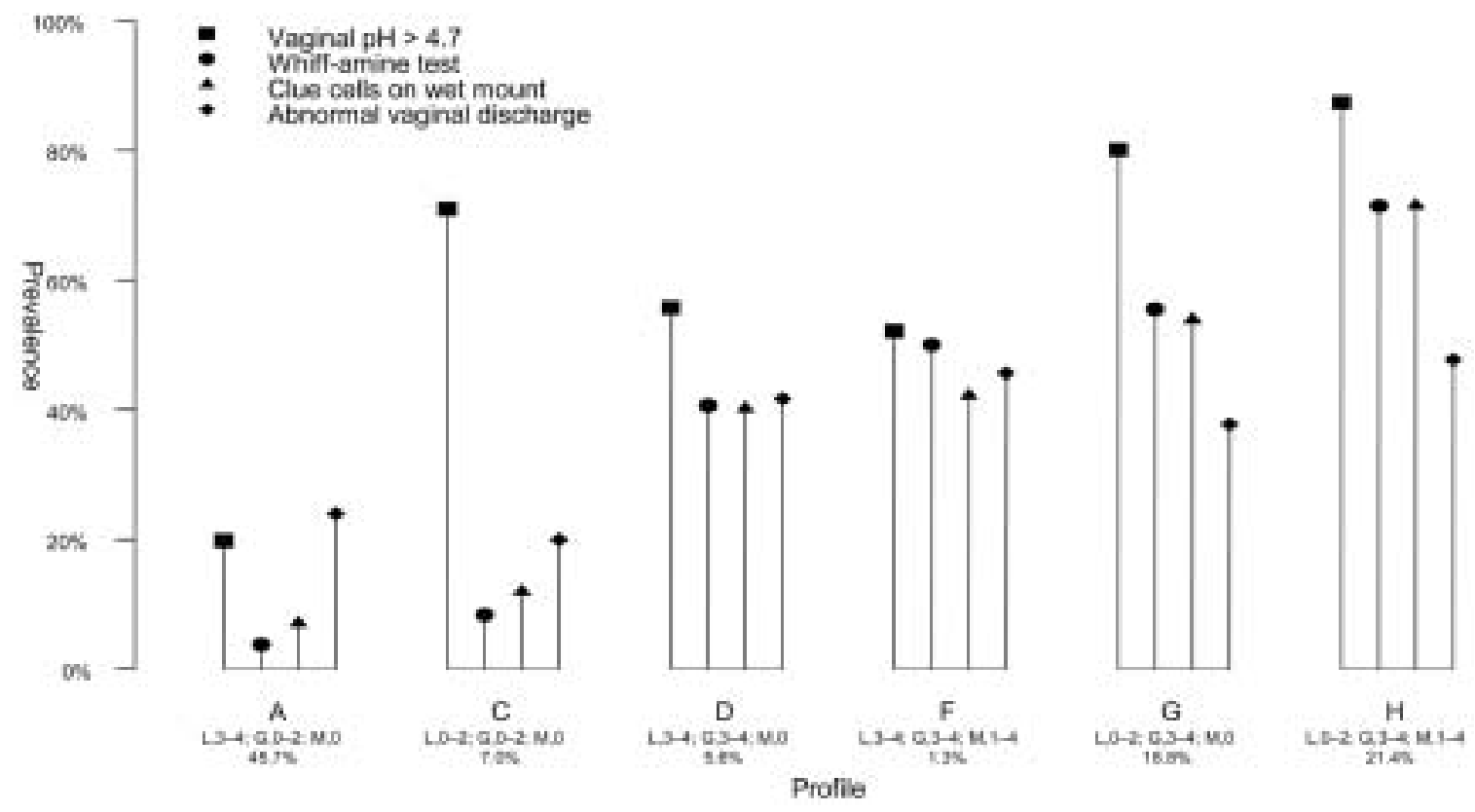

Figure I Prevalence of clinical signs by morphotypic profile 
met the clinical definition of BV. Thus conditions diagnosed as $\mathrm{BV}$ have the potential to differ greatly depending on the diagnostic method employed.

Our analysis did not assess host factors related to BV. Optimal prediction of adverse outcomes requires information on both vaginal microflora and host responses. Because this data set has few outcomes (most notably no obstetric data), no other vaginal symptoms, and limited behavioral factors, we were unable to assess the utility of these profiles in predicting adverse outcomes. Finally, the fact that all the women in this study had risk factors for acquiring HIV and other STDs may limit the generalizability of our findings.

Our large, multi-site, prospective study evaluated women using standardized protocols for both clinical and lab BV diagnostic strategies and centralized readings of all Gram stains. The laboratory diagnosis of $\mathrm{BV}$ (interpretation of the Gram stain by the Nugent score) is considered the gold standard in most research settings but is difficult to conduct in clinical settings.

Additionally, the Nugent scoring system conflates women with potentially very different microflora in a single category. This study takes the Gram stain data beyond the Nugent score, highlighting profiles such as $\mathrm{G}, \mathrm{H}$, and $\mathrm{F}$. One can have a Nugent score of 7 to 10 with or without the presence of Mobiluncus, and some patients have high numbers of Gardnerella and Mobiluncus while retaining Lactobacillus-like organisms. This study also emphasizes the occurrence of large numbers of women with abnormal or intermediate flora. These points serve to remind us that BV is not a pure, homogenous entity and is difficult to diagnose by any current method.

BV has been an elusive vaginal condition for both researchers and clinicians. The response to therapy is not uniform, the correlation with complications is variable, randomized control trials designed to eradicate $\mathrm{BV}$ and prevent complications have resulted in contradictory results, and discrepancies exist between Amsel and Nugent diagnostic methods.

Our results represent an attempt to break down the patterns of abnormal vaginal flora into more meaningful categories. Our data point to the need for further research using the morphotypic profiles in other samples of women and for examination of their association with obstetric outcomes. It is promising that by further elucidating the morphotypic make-up of the vaginal flora, beyond what is possible with the current data available for this analysis, a diagnostic system can be articulated that better correlates with risk factors for and outcomes of abnormalities in vaginal flora.

\section{REFERENCES}

1. Eschenbach DA, Hillier S, Critchlow C, et al. Diagnosis and clinical mainfestations of bacerial vaginosis. Am J Obstet Gynecol 1988;158:819-28

2. Hallén A, Påhlson C, Forsum U. Bacterial vaginosis in women attending STD clinics: diagnostic criteria and prevalence of Mobiluncus spp. Genitorium Med 1987;63:836-9

3. Paavonen J, Teisala K, Heinonen PK, et al. Microbiological and histopathological findings in acute pelvic inflammatory disease. $\mathrm{Br} J$ Obstet Gynaecol 1987;94:454-60

4. McDonald HM, O'Loughlin JA, Jolley PA, et al. Prenatal microbiological risk factors associated with preterm birth. Br J Obstet Gynaecol 1992;99: 190-6
5. Sewankambo N, Gray R, Waiver MJ, et al. HIV1 infection associated with abnormal vaginal flora morphology and bacterial vaginosis. Lancet 1997; 350:546-50

6. Martin HL, Richardson BA, Nyange PM, et al. Vaginal lactobacilli, microbial flora, and risk of human immunodeficiency virus type 1 and sexually transmitted disease acquisition. J Inf Dis 1999;180(6):1863-8

7. American College of Obstetricians and Gynecologists. ACOG Technical Bulletin: Vaginitis. Technical Bulletin number 226. July 1996 
8. Nugent RP, Krohn MA, Hillier SL. Reliability of diagnosing bacterial vaginosis is improved by a standardized method of gram stain interpretation. J Clin Microbiol 1991;29(2):297-301

9. McGregor JA, Hager WD, Gibbs RS, et al. Assessment of office-based care of sexually transmitted diseases and vaginitis and antibiotic decision-making by obstetrician-gynecologists. Infect Dis Obstet Gynecol 1998;6:247-51

10. Spiegel CA, Amsel R, Holmes KK. Diagnosis of bacterial vaginosis by direct gram stain of vaginal fluid. J Clin Microbiol 1983;18(1):170-7

11. Mastrobattista JM, Bishop KD, Newton ER. Wet smear compared with gram stain diagnosis of bacterial vaginosis in asymptomatic pregnant women. Obstet Gynecol 2000;96(4):504-6
12. Smith DK, Warren Dl, Vlahov D, et al. Design and baseline participant characteristics of the Human Immunodeficiency Virus Epidemiology Research (HER) Study: a prospective cohort study of human immunodeficiency virus infection in U.S. women. Am J Epi 1997;146(6):459-69

13. Amsel R, Totten PA, Speigel CA, et al. Nonspecific vaginitis. Diagnostic criteria and microbial and epidemiologic associations. Am J Med 1983;74(1):14-22

14. Jamieson D, Duerr A, Klein RS, et al. Longitudinal analysis of bacterial vaginosis: findings from the HIV Epidemiology Research Study. Obstet Gynecol 2001;98(4):656-63 


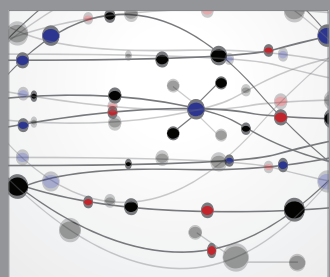

The Scientific World Journal
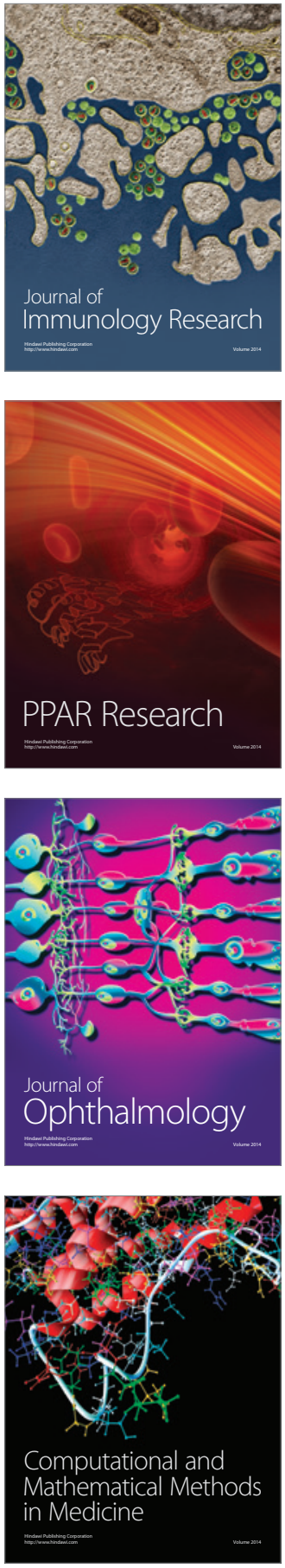

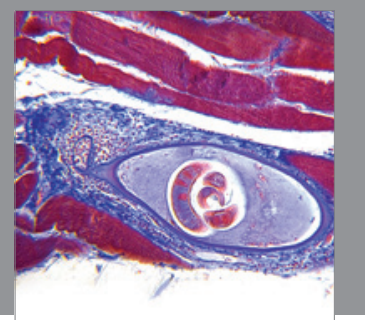

Gastroenterology

Research and Practice
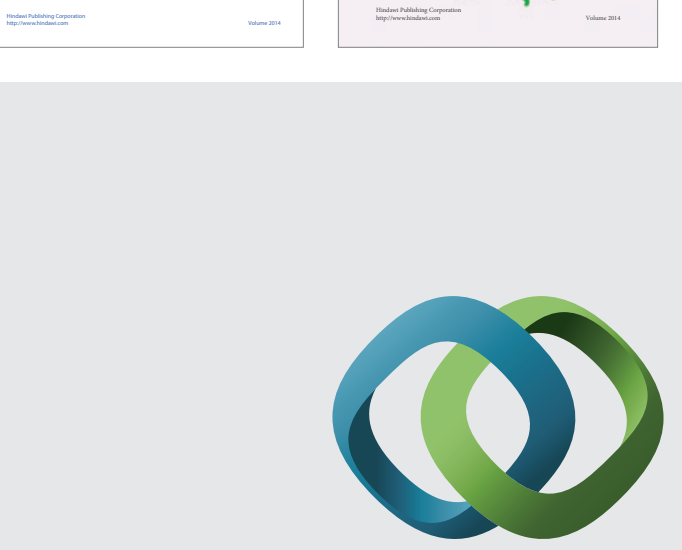

\section{Hindawi}

Submit your manuscripts at

http://www.hindawi.com
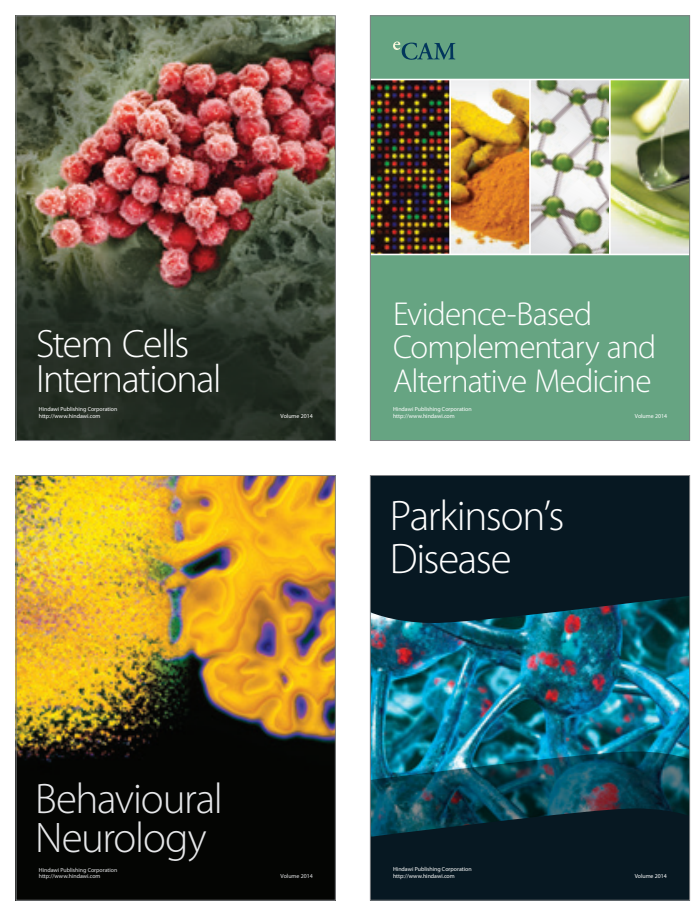

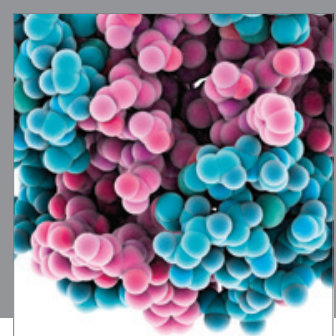

Journal of
Diabetes Research

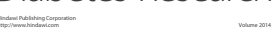

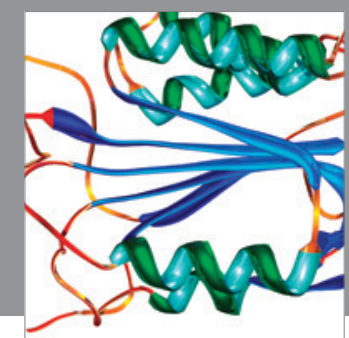

Disease Markers
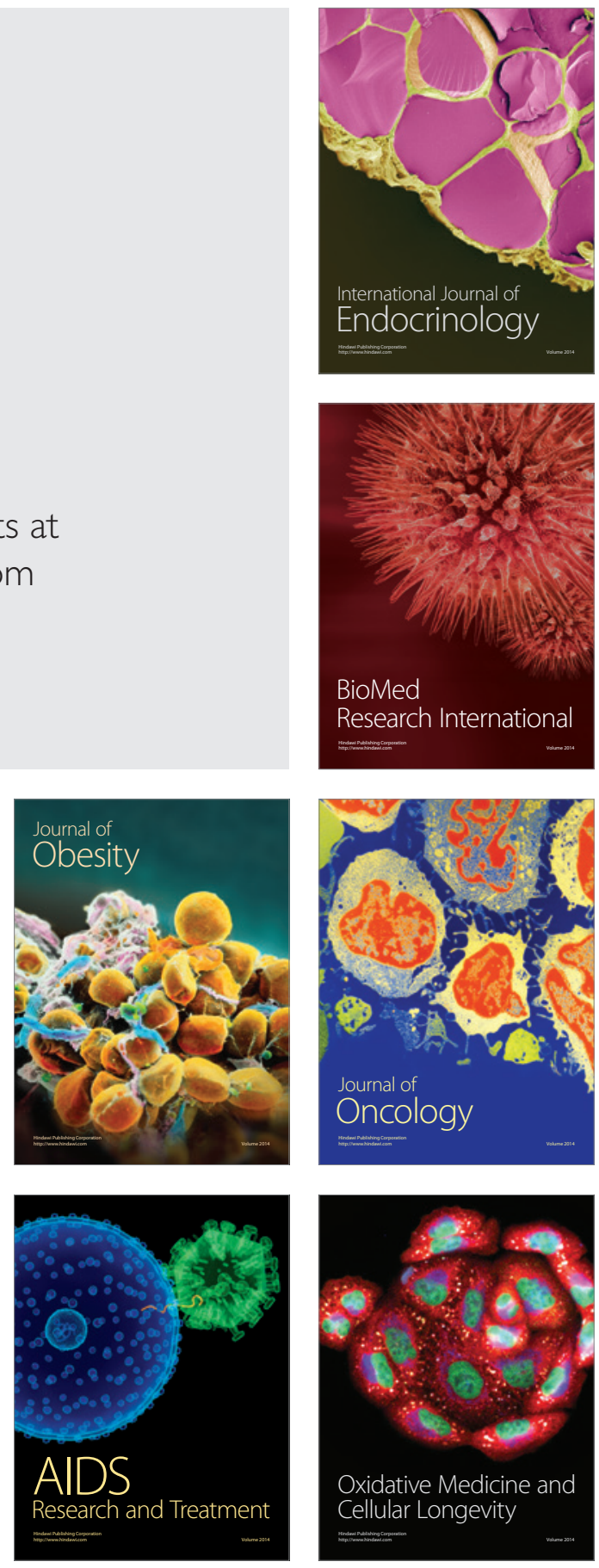